\title{
APTITUD COMBINATORIA Y HETEROSIS EN VARIEDADES DE GERBERA (Gerbera $\times$ hybrida)
}

\author{
COMBINING ABILITY AND HETEROSIS IN GERBERA \\ (Gerbera $\times$ hybrida) VARIETIES
}

\section{Azucena Rivera-Colín', Jaime Mejía-Carranza*, Luis M. Vázquez-García', Elizabeth Urbina-Sánchez ${ }^{1}$ y Marithza G. Ramírez-Gerardo²}

\author{
'Universidad Autónoma del Estado de México, Centro Universitario Tenancingo, Tenancingo, Estado de México, México. ${ }^{2}$ Tecnológico de Estudios \\ Superiores de Villa Guerrero, División de Ingeniería en Innovación Agrícola Sustentable, Villa Guerrero, Estado de México, México. \\ *Autor para correspondencia (jmejiac@uaemex.mx)
}

\section{RESUMEN}

Los cultivos que cuentan con un número considerable de variedades cultivadas, como la gerbera (Gerbera $x$ hybrida), pueden ser evaluados mediante experimentos de cruzas dialélicas para la formación de nuevos híbridos. El objetivo de la presente investigación fue estimar la aptitud combinatoria general (ACG) de seis variedades de gerbera y la aptitud combinatoria específica (ACE) de sus cruzas dialélicas, así como la heterosis en componentes de rendimiento y calidad de flores. Se empleó el método 1 de progenitores y sus cruzas $F_{1}$ directas y recíprocas con el modelo II de efectos aleatorios de Griffing. La evaluación se realizó bajo un diseño experimental de bloques completos al azar con cuatro repeticiones. El análisis estadístico detectó diferencias significativas $(\mathrm{P} \leq 0.01)$ dentro y entre progenitores y cruzas, así como en las interacciones, lo que evidenció amplia variabilidad entre progenitores y efectos maternos significativos por herencia citoplásmica. Los mayores efectos de ACG $(\mathrm{P} \leq 0.01)$ para rendimiento de tallos florales lo tuvieron los progenitores IGL, MG2 y FAS, mientras que los mayores efectos de ACE los mostraron las cruzas FAS $\times$ JES, ALC $\times$ IGL y DIN $\times$ IGL. Se encontraron efectos positivos de heterosis para rendimiento en las cruzas FAS $\times I G L$, JES $\times$ IGL, ALC $\times$ IGL, DIN $\times$ IGL, MG2 × IGL y FAS $\times$ MG2. Los resultados sugieren que Igloo y Fascination pueden ser utilizados como progenitores superiores en programas de cruzamiento para desarrollo de nuevas variedades de gerbera. Los valores altamente significativos de ACG para rendimiento de flor indican la importancia relativa de la acción génica aditiva sobre los efectos no aditivos.

Palabras clave: Gerbera $\times$ hybrida, aptitud combinatoria general, aptitud combinatoria específica, cruzas dialélicas, heterosis, rendimiento, calidad de flor.

\section{SUMMARY}

Crops that have a considerable number of cultivated varieties such as gerbera (Gerbera $\times$ hybrida) can be evaluated by diallel cross experiments for the formation of new hybrids. The aim of this research was to estimate the general combining ability (GCA) of six varieties of gerbera and the specific combining ability (SCA) of their diallel crosses and the heterosis on yield components and flower quality. The Griffing method 1 of parents and their direct and reciprocal $F_{1}$ crosses was used with model II of random effects. The evaluation was carried out under a randomized complete blocks experimental design with four replications. The statistical analysis detected significant differences $(P \leq 0.01)$ within and between parents and crosses, as well as in the interactions, which showed wide variability between parents and significant maternal effects due to cytoplasmic inheritance. The greatest effects of GCA $(P \leq 0.01)$ for yield of floral stems were obtained by the IGL, MG2 and FAS progenitors, while the greatests effects of SCA were shown by crosses FAS $\times$ JES, ALC $\times$ IGL and DIN $\times$ IGL. Positive effects of heterosis for yield were found in the crosses FAS $\times I G L, J E S \times I G L, A L C \times I G L, D I N \times I G L, M G 2 \times I G L$ and FAS $\times$ MG2. The results suggest that Igloo and Fascination can be used as superior parents in breeding programs for the development of new gerbera varieties. Highly significant GCA values for flower yield indicate the relative importance of additive gene action over non-additive effects.

Index words: Gerbera $\times$ hybrida, general combining ability, specific combining ability, diallel crosses, heterosis, yield, flower quality.

\section{INTRODUCCIÓN}

La floricultura en México, con más de 200 especies cultivadas, demanda una fuerte inversión, principalmente para la adquisición de material vegetativo, ya que las nuevas variedades son casi en su totalidad desarrolladas por empresas extranjeras (Gómez-Gómez, 2010); en particular, el cultivo de gerbera (Gerbera $\times$ hybrida) demanda hasta $80 \%$ de inversión para la adquisición de material vegetativo, el cual es desarrollado principalmente por hibridación, técnica iniciada en esta especie desde hace más de 100 años por Robert Jameson, con las especies Gerbera jamesonii Bolus y Gerbera viridifolia Sch. (Harding et al., 1981a, 1981b; Singh et al., 2011). Una alternativa para disminuir costos de importación de material vegetativo de gerbera en México es utilizar la variabilidad genética existente, prerrequisito indispensable en un programa de mejoramiento genético para desarrollar materiales propios (Rimieri, 2017). En este sentido, el aprovechamiento de componentes como la aptitud combinatoria, con la estimación de parámetros genéticos, permite la identificación de progenitores y cruzamientos superiores (Sprague y Tatum, 1942).

La aptitud combinatoria general (ACG) y la aptitud combinatoria específica (ACE) predicen el comportamiento de los parentales y su desempeño en combinaciones híbridas, respectivamente (Sprague y Tatum, 1942). La 
ACG se considera asociada a la acción génica de tipo aditivo y la ACE a la de tipo no aditivo (Griffing, 1956). La ACG y la ACE pueden ser evaluadas mediante un sistema de cruzamientos dialélicos, en el que una opción es realizar todos los posibles cruzamientos entre varios parentales (Dickinson y Jinks, 1956; Griffing, 1956; Hayman, 1958; Kempthorne, 1956). Se han propuesto diferentes métodos para el análisis de diseños dialélicos (Griffing, 1956; Hayman, 1958; Kempthorne, 1956), de los cuales se distinguen cuatro métodos experimentales de cruces dialélicos, mismos que varían dependiendo de la inclusión o no de los progenitores y los cruces recíprocos de las primeras generaciones filiales $\left(F_{1}\right)$ durante la evaluación.

Un aspecto importante del desempeño de los progenitores es el efecto heterótico de superioridad que éstos puedan expresar en sus híbridos para ser utilizados agronómicamente (Gutiérrez et al., 2002). De acuerdo con la gaceta oficial de los derechos de obtentor de variedades vegetales (SNICS, 2017), se tienen para gerbera 56 solicitudes de registro de nuevas variedades y si se consideran las que ya cuentan con un título de obtentor y las de dominio público, existen un poco más de 100 variedades en el mercado mexicano, las cuales representan una fuente de variabilidad genética que puede ser aprovechada en programas de hibridación para el desarrollo de nuevos materiales. Por lo anterior, el objetivo de la presente investigación fue estimar la ACG y ACE de seis variedades comerciales sobresalientes de gerbera y de sus cruzas dialélicas respectivamente, así como la heterosis en variables de rendimiento y calidad de flores mediante el método I de Griffing con el modelo II de efectos aleatorios.

\section{MATERIALES Y MÉTODOS}

\section{Material vegetal}

Para la obtención de las cruzas dialélicas se utilizaron seis variedades de gerbera preseleccionadas por estabilidad, calidad y rendimiento (Cuadro 1), las cuales fueron cultivadas en las instalaciones de la empresa Servicios Integrales de Horticultura Ornamental S. A. de C. V., ubicada en Villa Guerrero, Estado de México, a $18^{\circ} 54^{\prime}$ 58" latitud N y $99^{\circ} 38^{\prime} 37^{\prime \prime}$ longitud $\mathrm{O}$.

\section{Formación de híbridos y su manejo en etapa de plántula}

Los cruzamientos se realizaron entre progenitores femeninos con anteras inmaduras y progenitores masculinos con antesis completa y polen abundante. El capítulo del progenitor femenino fue emasculado con pinzas y polinizado con polen del progenitor masculino y cubierto con bolsas de tela de fibra textil "pellón" para evitar contaminación con polen extraño. Las semillas $F_{1}$ cosechadas a madurez fisiológica fueron sembradas, a razón de una semilla por cavidad, en charolas de germinación de 45 cavidades con sustrato de turba (Premier Peat moos ${ }^{\circledR}$ ) y agrolita $2: 1$ ( $/ \mathrm{v}$ ) hidratado al 100 $\%$ y mantenidas a temperatura de $21 \pm 3{ }^{\circ} \mathrm{C}$ y humedad relativa de $70 \%$.

Para aprovechar mejor el material generado, y de acuerdo con la experiencia en el laboratorio, las plántulas de cada cruza utilizadas para estimar ACG y ACE se preseleccionaron primero por su crecimiento vigoroso con

Cuadro 1. Origen y características de variedades de Gerbera $\times$ hibrida utilizadas en el estudio.

\begin{tabular}{lccccccccc}
\hline Variedad & CC & O & Ccap & Cd & Tcap & $\begin{array}{c}\text { Ro } \\
\text { (tallos/mmes) }\end{array}$ & VF (d) & DC (cm) & AP (cm) \\
\hline Igloo (IGL) & Th & H & Bl & V & Sm & 22 & 12 & 12 & 60 \\
Fascination (FAS) & Th & H & Rs & N & Sm & 20 & 14 & 12 & 60 \\
Alcatraz (ALC) & Th & H & Rj & V & Sm & 17 & 12 & 11 & 58 \\
Dino (DIN) & Tn & H & Am & V & Sm & 16 & 10 & 11 & 60 \\
Jessy (JES) & B & I & Nr & N & Sm & 17 & 10 & 11 & 60 \\
MG24 (MG2) & B & I & Fs & N & S & 17 & 17 & 11 & 58 \\
\hline
\end{tabular}

CC: casa comercial, Tn: Terra Nigra, B: Bindisirio; O: origen. H: Holanda, I: Italia, Ccap: color de capítulo, Bl: blanco, Rs: rosa, Nr: naranja, Am: amarillo, Rj: rojo, Fs: fiusha, Cd: color de disco, N: negro, V: verde, Tcap: tipo de capítulo, S: Sencillo, Sm: semidoble, Ro: rendimiento, VF: vida en florero, DC: diámetro de capítulo, AP : altura de pedúnculo. 
formación de tres o más pares de hojas verdaderas (Leffring, 1973) y después, por su buena capacidad organogénica en cultivo in vitro, criterios que son indispensables para el aprovechamiento y la propagación comercial de esta especie. Plántulas de $20 \mathrm{~cm}$ de altura de cada uno de los híbridos seleccionados se trasplantaron a macetas de 20 $L$ con $40 \%$ de tezontle rojo en la base y $60 \%$ de la mezcla turba:agrolita 2:1 ( $\mathrm{v} / \mathrm{v})$ en la parte superior.

\section{Evaluación experimental $y$ variables evaluadas}

Las macetas se establecieron bajo un diseño de bloques completos al azar con cuatro repeticiones. Los tratamientos fueron las variedades y la unidad experimental fue una planta por maceta. Se midieron las variables rendimiento (número de tallos florales $/ \mathrm{m}^{2} / \mathrm{mes}$ ); altura del pedúnculo $(\mathrm{cm})$, desde la base hasta el receptáculo del capítulo; diámetro del capítulo completamente abierto $(\mathrm{cm})$, medido ecuatorialmente a borde de lígulas; vida en florero a temperatura ambiente en una solución de $\mathrm{NaClO} 40$ ppm, medida en días, de acuerdo con la presencia de pistilos con polen abundante del exterior al interior del disco, y conservación de turgencia, forma y color de capítulos (De Jong, 1978).

\section{Análisis estadístico}

Se realizó un análisis de varianza y un análisis genético de las $\mathrm{P}^{2}$ cruzas formadas conforme al método I y modelo II de efectos aleatorios (Griffing, 1956). Para la estimación de la ACG y ACE sólo se evaluaron las $\mathrm{P}(\mathrm{P}-1) / 2$ combinaciones de las cruzas directas.

El análisis de los datos se realizó con el programa de cómputo InfoStat (Di Rienzo et al., 2011). Las estimaciones de heterosis $(H)$ se realizaron con base en la fórmula $H=$ $\left.\left[\left(F_{1}-V P M\right) / V P M\right)\right] \times 100$, donde: $F_{1}$ es el valor promedio de la cruza y VPM es el valor promedio de los progenitores (Reyes, 1985). Para obtener las significancias estadísticas entre los porcentajes de heterosis obtenidos por las cruzas, se realizó un análisis de varianza para cada variable $y$, donde el modelo detectó diferencias, se utilizó la prueba de comparación de medias de Tukey $(P \leq 0.05)$. Con base en la proporción relativa de los cuadrados medios de los efectos de ACG y ACE se determinó la contribución relativa de los efectos aditivos y no aditivos de las variables en estudio. Los efectos de ACG y ACE se probaron mediante la prueba de comparación de medias de Tukey $(P \leq 0.05)$.

Los valores superiores de las medias de progenitores y cruzas de las variables en estudio fueron aquellos que superaron al valor promedio de las medias más el error estándar $(\mu+E S)$. Como criterio de selección de las mejores cruzas se utilizó el valor del rendimiento superior a la media del mismo carácter más su error estándar.

\section{RESULTADOS Y DISCUSIÓN}

El desempeño de todos los progenitores y cruzas en rendimiento, altura de pedúnculo, diámetro de capítulo y vida de florero fue variable y con diferencias significativas en los parámetros medidos tanto dentro, como entre progenitores y sus cruzas (Cuadro 2), lo que sugiere amplia variabilidad genética entre progenitores.

Resultados similares en gerbera se han reportado en trabajos afines para forma del capítulo, número de tallos por planta, índice de área foliar y número de hojas por planta (Cardoso et al., 2009; Kumari et al., 2011; Senapati et al., 2013). Con respecto a rendimiento dentro de progenitores, sólo uno de ellos (FAS) fue superior significativamente a la media, en tanto que dentro de cruzas, nueve de 15 presentaron valores significativamente superiores a la media. En estas cruzas destacó FAS con mayor número de participaciones como progenitor. Similarmente, para diámetro de capítulo, dentro de progenitores, sólo el progenitor IGL fue superior significativamente a la media, en tanto que dentro de cruzas, casi la mitad (siete de 15) fueron superiores significativamente a la media, donde JES destacó como el progenitor con intervención en más cruzas.

En cuanto a vida de florero, cuatro de los seis progenitores mostraron valores superiores y significativos a la media; sin embargo, en las cruzas sólo dos de las 15 (DIN $\times$ IGL y MG2 × IGL) fueron superiores significativamente a la media, las cuales mostraron heterosis. La altura de planta en la mayoría de los progenitores fue superior significativamente a la media; no obstante, en las cruzas sólo cuatro de 15 fueron estadísticamente superiores. Los resultados anteriores indican que los progenitores participantes en cruzas sobresalientes pueden ser utilizados en programas de formación de híbridos intervarietales o como nuevas líneas con uno o más de los atributos considerados. Adicionalmente, también se identificaron cruzas específicas (JES $\times$ ALC, ALC $\times$ IGL y MG2 × IGL) que mostraron valores superiores a su respectiva media hasta en tres de las variables evaluadas.

El análisis de varianza del dialélico detectó diferencias altamente significativas $(P \leq 0.01)$ en las fuentes de variación para las cuatro variables evaluadas, donde estuvieron involucrados los progenitores y las cruzas directas y recíprocas (Cuadro 3).

Las diferencias altamente significativas en la interacción progenitores $\times$ cruzas revelaron, en algunos casos, efectos 
Cuadro 2. Medias de Rendimiento (Ro), vida en florero (VF), diámetro de capítulo (DC) y altura de pedúnculo (AP) de seis progenitores y 15 híbridos $F_{1}$ de gerbera.

\begin{tabular}{|c|c|c|c|c|}
\hline Genotipos & Ro (tallos/m²/mes) & $V F(d)$ & $\mathrm{DC}(\mathrm{cm})$ & $\mathrm{AP}(\mathrm{cm})$ \\
\hline \multicolumn{5}{|l|}{ Progenitores } \\
\hline FAS & $22.21^{\dagger}$ & $13.96^{t+}$ & 11.29 & $64.44^{++}$ \\
\hline JES & 21 & $14.37^{++}$ & 12.07 & $63.31^{+}$ \\
\hline ALC & 21.14 & $13.71^{+\dagger}$ & 11.59 & $63.25^{+}$ \\
\hline DIN & 20.62 & 12.5 & 11.82 & 63.06 \\
\hline MG2 & 20.71 & $12.92^{+}$ & 11.94 & $63.41^{+}$ \\
\hline IGL & 20.04 & 12.75 & $12.23^{++}$ & $66.16^{++}$ \\
\hline \multicolumn{5}{|l|}{ Cruzas } \\
\hline FAS $\times$ JES & $24.2^{++}$ & 12.45 & 11.98 & 63.18 \\
\hline$F A S \times A L C$ & 21.99 & 12.38 & $12.27^{++}$ & 62.98 \\
\hline FAS $\times$ DIN & $22.34^{++}$ & 12.32 & $12.09^{+}$ & 60.78 \\
\hline FAS $\times$ MG2 & $22.6^{++}$ & 11.92 & 11.92 & 62.03 \\
\hline$F A S \times I G L$ & $22.3^{++}$ & 11.94 & 11.94 & 61.42 \\
\hline $\mathrm{JES} \times \mathrm{ALC}$ & $22.46^{++}$ & 13.05 & $12.32^{++}$ & $64.03^{++}$ \\
\hline JES $\times$ DIN & $22.19^{+}$ & 12.72 & $12.72^{+t}$ & 61.03 \\
\hline JES $\times$ MG2 & 21.74 & 12.46 & $12.46^{+t}$ & 63.01 \\
\hline$J E S \times I G L$ & 21.22 & 10.63 & 10.63 & 60.93 \\
\hline$A L C \times D I N$ & $22.12^{+}$ & 11.96 & 11.5 & $64.97^{+t}$ \\
\hline $\mathrm{ALC} \times \mathrm{MG} 2$ & 20.89 & 11.3 & 11.3 & 60.95 \\
\hline$A L C \times I G L$ & $23.47^{++}$ & 12.55 & $12.49^{++}$ & $66.23^{+1}$ \\
\hline $\mathrm{DIN} \times \mathrm{MG} 2$ & $22.22^{+}$ & 11.2 & 11.2 & 60.53 \\
\hline$D I N \times I G L$ & $22.35^{+t}$ & $14.95^{++}$ & 12 & $63.23^{+}$ \\
\hline$M G 2 \times I G L$ & 21.45 & $13.39^{++}$ & $13.39^{+t}$ & 60.93 \\
\hline Media $(\mu)$ & 21.87 & 12.64 & 11.96 & 62.85 \\
\hline Error estándar (ES) & 0.21 & 0.23 & 0.13 & 0.38 \\
\hline
\end{tabular}

$\mu$ : media, ES: error estándar, ${ }^{+}$: mayor que $\mu+E S,{ }^{\text {t+: }}$ : mayor que $\mu+2 E S$.

heteróticos de las cruzas, en específico para rendimiento; sin embargo, para vida de florero el comportamiento de la mayoría de los progenitores fue superior a casi todas las cruzas. Las diferencias altamente significativas en la interacción cruzas directas $\times$ cruzas recíprocas sugieren efectos maternos por herencia citoplásmica u otra influencia del progenitor femenino (Mendoza-Elos et al., 2003), lo que deberá estudiarse más a detalle para dirigir las cruzas.

Los efectos de la ACG presentaron valores altamente significativos para rendimiento y vida de florero (Cuadro 4), lo que resalta la importancia relativa de la acción génica aditiva sobre los efectos no aditivos en dichas variables.

En los casos donde el efecto de ACG fue mayor al de
ACE es recomendable hacer selección de genotipos con dichas cualidades y utilizar al menos a uno de ellos como progenitor en programas de cruzas simples, ya que según Reyes et al. (2004), una cruza simple con al menos uno de sus progenitores con alta ACG puede garantizar altos valores en el rasgo evaluado. Valores significativos de ACG en componentes de rendimiento también han sido reportados para gerbera (Harding et al., 1981b) y otros cultivos como girasol (Helianthus annuus L.) (Kaya y Atakisi, 2004; Machikowa et al., 2011), tomate de cáscara (Physalis ixocarpa Brot.) (Camposeco et al., 2015), chile (Capsicum sp.) (Gomide et al., 2003) y maíz (Zea mays L.) (Reyes et al., 2004), lo que demuestra que la acción génica aditiva constituye uno de los componentes genéticos más importantes del rendimiento en diferentes especies. Contrariamente, Sánchez-Hernández et al. (2011) en 
Cuadro 3. Cuadrados medios del análisis de varianza del diseño dialélico de seis progenitores de gerbera y sus 15 cruzas directas y 15 recíprocas.

\begin{tabular}{|c|c|c|c|c|c|}
\hline Fuente de variación & G. L. & Ro & VF & DC & $\mathrm{AP}$ \\
\hline Bloques & 3 & $1.57 *$ & $0.18 *$ & $0.37 * *$ & $0.65 * *$ \\
\hline Progenitores y Cruzas & 35 & $4.07 * \star$ & $5.50 * *$ & $1.64 * *$ & $12.16 * *$ \\
\hline Progenitores & 5 & $2.05 * \star$ & $2.22 * *$ & $0.47 * *$ & $14.77 * *$ \\
\hline Cruzas directas & 14 & $2.68 * \star$ & $4.51 * *$ & 1.81 ** & $11.81 * *$ \\
\hline Cruzas recíprocas & 14 & $4.58 * \star$ & $6.95 * *$ & $2.07 * \star$ & $13.47 * *$ \\
\hline Progenitores $\times$ Cruzas & 1 & $586.01 * *$ & $2200.99 * *$ & $128.60 * *$ & $2702.43 * *$ \\
\hline C. directas $\times$ C. recíprocas & 1 & $52.85 * \star$ & $0.71 * \star$ & $4.97 \star \star$ & $15.46 * *$ \\
\hline Error & 175 & 0.41 & 0.06 & 0.03 & 0.09 \\
\hline Total & 213 & & & & \\
\hline C.V. & & 2.94 & 1.88 & 1.50 & 0.49 \\
\hline
\end{tabular}

Cuadro 4. Cuadrados medios del análisis de varianza para ACG y ACE de seis progenitores y 30 cruzas de gerbera para rendimiento y calidad de flor.

\begin{tabular}{llllll}
\hline Fuente de variación & G. L. & Ro & VF & DC & AP \\
\hline Bloques & 3 & 0.87 & 0.05 & 0.07 & 44.37 \\
ACG & 5 & $6.40 * \star$ & $4.22 * \star$ & 7.06 & 247.44 \\
ACE & 9 & 0.61 & $4.69 * *$ & $6.74 * *$ & $265.50 * *$ \\
Error & 42 & 1.00 & 0.03 & 0.03 & 64.99 \\
Total & 59 & & & &
\end{tabular}

G. L.: grados de libertad, Ro: rendimiento, VF : vida en florero, DC: diámetro de capítulo, AP : altura de pedúnculo, ACG: aptitud combinatoria general, ACE: aptitud combinatoria específica, *: significativo a 0.05 de probabilidad, **: significativo a 0.01 de probabilidad.

calabacita (Cucurbita pepo L.) reportaron que la acción génica no aditiva fue más importante en componentes del rendimiento de frutos por planta, largo de fruto y rendimiento por planta, por lo que se deben hacer evaluaciones para dirigir el mejoramiento genético de acuerdo con la especie y el carácter de interés.

Por otra parte, los efectos de ACE mostraron valores altamente significativos en vida de florero, diámetro de capítulo y altura de pedúnculo, lo que da importancia a desviaciones de la varianza aditiva como dominancia, sobredominancia o epistasis y sugieren un efecto complementario de los progenitores involucrados en particular en las cruzas sobresalientes en dichas variables, que pudieran ser utilizados específicamente para formar nuevas líneas. En estos casos, hibridaciones con progenitores específicos resultarían de utilidad para mejorar el carácter e incluso para generar más variabilidad genética y poder hacer selección. Resultados de ACE en $H$. annuus, especie de la misma familia que la gerbera, mostraron valores estadísticamente significativos para altura de planta (Laureti y Del Gatto, 2001).

De acuerdo con Cervantes et al. (2011), es recomendable aprovechar primero la varianza genética aditiva por selección de progenitores con buena ACG y después la varianza no aditiva por hibridación para cruzas con buena ACE; sin embargo, esto depende de la proporción que representa la varianza aditiva y la de dominancia de la población estudiada con respecto a la variación genética total (Reyes et al., 2004), aunque en un programa de mejoramiento genético de gerbera, dependiendo de los objetivos específicos, se pueden considerar tanto los efectos de ACG como los efectos de ACE.

La estimación de los efectos de ACG de los progenitores mostró efectos positivos y significativos para rendimiento en las variedades FAS, MG2 e IGL (Cuadro 5), lo que indica que estas variedades tienen una alta contribución en la expresión del rendimiento y que los efectos aditivos son los más importantes; por lo tanto, pudieran incluirse como progenitores contribuyentes de alelos superiores 
para rendimiento en programas de formación de nuevas variedades mediante métodos de hibridación o formación de sintéticos, cuyo desempeño para un carácter determinado podría verificarse mediante una evaluación de la generación $F_{2}$ (De la Cruz-Lázaro et al., 2010).

Según la magnitud de los efectos de ACE, las mejores cruzas con valores positivos y significativos fueron FAS $\times$ JES y ALC $\times$ IGL para rendimiento y DIN $\times$ IGL para vida de florero. De los seis progenitores que intervinieron en las cruzas, FAS e IGL mostraron efectos positivos y significativos de ACG, donde IGL repitió en dos cruzas, tanto para rendimiento como para vida de florero. Aun cuando los efectos de la ACG de los seis progenitores para vida de florero fueron negativos, se obtuvieron efectos positivos de la ACE en siete de las 15 cruzas; es decir, la interacción entre los progenitores fue buena. Resultados similares han sido reportados en maíz para rendimiento (Guillen-De la Cruz et al., 2009); sin embargo, Harding et al. (1981a) para vida de florero reportaron heredabilidades bajas en sentido estrecho en gerbera, lo que sugiere avance lento en la mejora. No hubo valores significativos para diámetro de capítulo ni para altura de pedúnculo. Las cruzas que presentaron efectos con valores positivos en las cuatro variables evaluadas fueron JES $\times$ ALC y ALC $\times$ IGL.

La estimación de la heterosis para rendimiento indicó un efecto aditivo de las variedades en 14 de las 15 cruzas, con excepción de FAS × DIN, cuyo valor fue negativo muy próximo a cero (Cuadro 6). Destacó para esta variable el progenitor IGL que participó en cinco (FAS $\times I G L, J E S \times I G L$, $A L C \times I G L, D I N \times I G L$ y MG2 × IGL) de las siete cruzas con mayor valor heterótico, lo que lo hace un progenitor superior,

Cuadro 5. Efectos de aptitud combinatoria general y específica para rendimiento (Ro), vida en florero (VF), diámetro de capítulo (DC) y altura de pedúnculo (AP) de seis variedades y 15 cruzas directas de gerbera.

\begin{tabular}{|c|c|c|c|c|}
\hline Genotipo & Ro (tallos $/ \mathrm{m}^{2} / \mathrm{mes}$ ) & $V F(d)$ & $\mathrm{DC}(\mathrm{cm})$ & $\mathrm{AP}(\mathrm{cm})$ \\
\hline & \multicolumn{4}{|c|}{ Efectos de aptitud combinatoria general } \\
\hline FAS & $2.49 *$ & -0.05 & 0.03 & 2.54 \\
\hline JES & 1.09 & -0.05 & -0.05 & 8.92 \\
\hline ALC & 0.57 & -0.05 & 0.07 & 10.55 \\
\hline DIN & 0.72 & -0.02 & 0.02 & $16.14 *$ \\
\hline MG2 & $6.05 * *$ & -0.05 & 0.09 & 0.24 \\
\hline \multirow[t]{2}{*}{ IGL } & $6.41 * *$ & -0.04 & 0.37 & 2.56 \\
\hline & \multicolumn{4}{|c|}{ Efectos de aptitud combinatoria específica } \\
\hline FAS $\times$ JES & $2.54 * *$ & 0.04 & 0 & 0.45 \\
\hline FAS $\times$ ALC & 0.16 & -0.21 & 0.24 & 1.64 \\
\hline FAS $\times$ DIN & 0.56 & 0.1 & -0.01 & -2.28 \\
\hline$F A S \times M G$ & 0.36 & -0.7 & -0.03 & -1.65 \\
\hline $\mathrm{FAS} \times I G L$ & 0.43 & -0.04 & 0.03 & -1.20 \\
\hline $\mathrm{JES} \times \mathrm{ALC}$ & 0.3 & 0.52 & 0.28 & 3.06 \\
\hline JES $\times$ DIN & 0.09 & 0.56 & 0.6 & -1.67 \\
\hline $\mathrm{JES} \times \mathrm{MG} 2$ & -0.82 & -0.10 & 0.49 & -0.32 \\
\hline $\mathrm{JES} \times \mathrm{IGL}$ & -0.97 & -1.28 & -1.30 & -1.34 \\
\hline $\mathrm{ALC} \times \mathrm{DIN}$ & -0.15 & -1.25 & -0.66 & 3.67 \\
\hline$A L C \times M G$ & -1.85 & -1.44 & -0.71 & -0.98 \\
\hline$A L C \times I G L$ & $1.11 *$ & 0.44 & 0.52 & $5.36 *$ \\
\hline $\mathrm{DIN} \times \mathrm{MG} 2$ & -0.47 & -1.16 & -0.88 & -3.12 \\
\hline $\mathrm{DIN} \times I G \mathrm{I}$ & 0.04 & $3.23 * *$ & -0.05 & 0.64 \\
\hline MG2 × IGL & -1.33 & 1.27 & 1.5 & -2.28 \\
\hline
\end{tabular}

*: significativo a 0.05 de probabilidad, **: significativo a 0.01 de probabilidad. 
Cuadro 6. Heterosis de 15 cruzas con respecto al promedio de progenitores para rendimiento (Ro), vida en florero (VF), diámetro de capítulo (DC) y altura de pedúnculo (AP).

\begin{tabular}{lcrrr}
\hline \multirow{2}{*}{ Cruza } & Ro (tallos $\left./ \mathrm{m}^{2} / \mathrm{mes}\right)$ & VF $(\mathrm{d})$ & $\mathrm{DC}(\mathrm{cm})$ & $\mathrm{AP}(\mathrm{cm})$ \\
\cline { 2 - 4 } & \multicolumn{3}{c}{ Heterosis $(\%)$} & -1.73 \\
\hline FAS $\times$ JES & 5.07 & -18.80 & -3.53 & -3.60 \\
FAS $\times$ ALC & 1.26 & -11.05 & 7.06 & -3.88 \\
FAS $\times$ DIN & -0.39 & -6.53 & 6.03 & -0.09 \\
FAS $\times$ MG2 & 7.76 & -0.80 & 7.27 & -1.31 \\
FAS $\times$ IGL & 9.33 & -8.13 & 4.17 & -0.90 \\
JES $\times$ ALC & 5.26 & -9.18 & 3.16 & -3.52 \\
JES $\times$ DIN & 2.92 & -7.21 & 4.37 & -2.64 \\
JES $\times$ MG2 & -9.33 & 3.06 & -2.76 \\
JES $\times$ IGL & 3.63 & -7.13 & -2.85 & -2.32 \\
ALC $\times$ DIN & 10.09 & -5.59 & 5.70 & -1.94 \\
ALC $\times$ MG2 & 3.51 & -2.17 & -1.42 & -0.80 \\
ALC $\times$ IGL & 3.10 & -10.81 & -1.10 & -3.25 \\
DIN $\times$ MG2 & 9.88 & -12.85 & -6.76 & -0.98 \\
DIN $\times$ IGL & 8.52 & 8.74 & 0.70 & -3.48 \\
MG2 $\times$ IGL & 8.80 & -1.27 & 3.64 & \\
\hline
\end{tabular}

que de acuerdo con De la Cruz-Lázaro et al. (2010), desde el punto de vista comercial, pudiera ser de importancia por el vigor híbrido generado. En diámetro de capítulo, 10 de las 15 cruzas mostraron un efecto aditivo de heterosis de los progenitores involucrados, donde el progenitor FAS destacó también como un progenitor superior que participó en las tres cruzas con mayor valor heterótico (FAS $\times$ ALC, FAS $\times$ DIN y FAS $\times$ MG2); sin embargo, en las variables vida de florero y altura de pedúnculo no hubo efecto aditivo, con valores negativos en todas las cruzas, excepto en la cruza DIN $\times I G L$, lo que sugiere desviaciones de aditividad en combinaciones híbridas varietales específicas.

La amplitud de la heterosis para el rendimiento, que osciló de -0.39 a $10.09 \%$, sugiere un gradiente de divergencia genética entre los progenitores. De acuerdo con Romero et al. (2002), a mayor divergencia genética se presenta mayor heterosis, aunque también destacan que la ausencia de heterosis no necesariamente implica falta de divergencia. De igual manera, Gutiérrez et al. (2002) reportaron que la diferencia entre los valores de ACG y ACE aumenta a medida que la divergencia genética de los materiales se incrementa, hasta un cierto límite (Hallauer et al., 2010). En tres de las cruzas con el menor valor heterótico participó DIN como progenitor, lo que sugiere que éste, junto con los otros progenitores involucrados, pudiera compartir alelos comunes para el rendimiento de gerbera.

\section{CONCLUSIONES}

El desempeño de los progenitores de gerbera y cruzas en el análisis dialélico sugiere amplia variabilidad genética entre los progenitores. Los valores altamente significativos de ACG para rendimiento de flor indican la importancia relativa de la acción génica aditiva sobre los efectos no aditivos en dicha variable. Valores altamente significativos de ACE para vida de florero, diámetro de capítulo y altura de pedúnculo dan importancia a la varianza genética no aditiva, que pudo manifestarse en cruzas específicas y los valores positivos de heterosis, lo que permite identificar a algunas variedades como progenitores superiores que, desde el punto de vista comercial, pudieran ser de gran importancia por el vigor híbrido generado.

\section{AGRADECIMIENTOS}

Se agradece al Consejo Nacional de Ciencia y Tecnología (CONACYT) por el apoyo financiero otorgado y a la empresa Servicios Integrales de Horticultura Ornamental S. A. de C. V., por las facilidades brindadas para la realización del trabajo experimental. 


\section{BIBLIOGRAFÍA}

Camposeco M. N., V. Robledo T., L. A. Valdez A., F. Ramírez G., R. Mendoza V. y A. Benavides M. (2015) Estimación de la aptitud combinatoria en poblaciones de tomate de cáscara. Revista Mexicana de Ciencias Agrícolas 6:437-451

Cardoso R. D. L., M. F. Grando, S. M. S. Basso, M. I. Segeren, L. Augustin e M. Suzin (2009) Caracterização citogenética, viabilidade de pólen e hibridação artificial em gérbera. Horticultura Brasileira 27:4044, https://doi.org/10.1590/S0102-05362009000100008

Cervantes 0. F., G. García-De los Santos, A. Carballo C., D. Bergvinson, J. L. Crossa, M. Mendoza E., E. Andrio E., J. G. Rivera R. y E. Moreno M. (2011) Estimación de efectos genéticos relacionados con el vigor de la semilla y de la plántula en maíces tropicales mexicanos. Phyton 80:19-26.

De Jong J. (1978) Dry storage and subsequent recovery of cut gerbera flowers as an aid in selection for longevity. Scientia Horticulturae 9:389-397, https://doi.org/10.1016/0304-4238(78)90048-1

De la Cruz-Lázaro E., G. Castañón-Najera, N. P. Brito-Manzano, A. GómezVázquez, V. Robledo-Torres y A. J. Lozano R. (2010) Heterosis y aptitud combinatoria de poblaciones de maíz tropical. Phyton 79:11-17.

Di Rienzo J. A., F. Casanoves, M. G. Balzarini, L. González, M. Tablada y C. W. Robledo (2011) Infostat: Software Estadístico. Manual del Usuario. Versión 2011. Universidad Nacional de Córdoba. Córdoba, Argentina. $336 \mathrm{p}$

Dickinson A. G. and J. L. Jinks (1956) A generalised analysis of diallel crosses. Genetics 41:65-78.

Gómez-Gómez A. A. (2010) La situación de las flores de corte mexicanas dentro de la política comercial internacional de México. Tecsistecatl 2:1-30.

Gomide M. L., W. R. Maluf y L. A. A. Gomes (2003) Heterose e capacidade combinatória de linhagens de pimentão (Capsicum annuum L.). Ciência e Agrotecnologia, 27:1007-1015.

Griffing B. (1956) Concept of general and specific combining ability in relation to diallel crossing systems. Australian. Journal of Biological Sciences 9:463-493.

Guillen-de la Cruz P., E. de la Cruz-Lázaro, G. Castañon-Najera, R. Osorio-Osorio, N. P. Brito-Manzano, A. Lozano-del Río y U. López-Noverola (2009) Aptitud combinatoria general y específica de germoplasma tropical de maíz. Tropical and Subtropical Agroecosystems 10:101-107.

Gutiérrez del R. E., A. Palomo G., A. Espinoz B. y E. De la Cruz L. (2002) Aptitud combinatoria y heterosis para rendimiento de líneas de maíz en la Comarca Lagunera, México. Revista Fitotecnia Mexicana 25:271-277.

Hallauer A. R., M. J. Carena and J. B. Miranda Filho (2010) Quantitative Genetics in Maize Breeding. Springer. New York, N. Y., USA. 663 p.

Harding J., T. Byrne and R. L. Nelson (1981a) Heritability of cut-flower vase longevity in gerbera. Euphytica 30:653-657, https://doi. org/10.1007/BF00038793

Harding J., T. G. Byrne and R. L. Nelson (1981b) Estimation of heritability and response to selection for cut-flower yield in gerbera. Euphytica 30:313-322, https://doi.org/10.1007/BF00033992
Hayman B. I. (1958) The theory and analysis of diallel crosses. II. Genetics 43:63-85.

Kaya Y., and I. K. Atakisi (2004) Combining ability analysis of some yield characters of sunflower (Helianthus annuus L.). Helia 27:75-84, https://doi.org/10.2298/hel0441075y

Kempthorne 0. (1956) The theory of diallel cross. Genetics 41:451-459.

Kumari A., K. S. Patel and M. Choudhary (2011) Genetic variability studies in Gerbera. Research in Plant Biology 1:1-4.

Laureti D. and A. Del Gatto (2001) General and specific combining ability in sunflower (Helianthus annuus L.). Helia 24:1-16.

Leffring L. (1973) Flower production in gerbera: I. Correlations between shoot, leaf and flower formation in seedlings. Scientia Horticulturae 1:221-229, https://doi.org/10.1016/03044238(73)90034-4

Machikowa T., C. Saetang and K. Funpeng (2011) General and specific combining ability for quantitative characters in sunflower. Journal of Agricultural Science 3:91-95, https://doi. org/10.5539/jas.v3n1p91

Mendoza-Elos M., A. López-Benitez, A. Oyervides-García, G. MartínezZambrano, C. De León y E. Moreno-Martínez (2003) Herencia genética y citoplásmica de la resistencia a la pudrición de la mazorca de maíz (Zea mays L.) causada por Fusarium moniliforme Sheld. Revista Mexicana de Fitopatología 21: 267-271

Reyes C. P. (1985) Fitogenotecnia Básica y Aplicada. AGT Editor. México, D. F. 460 p.

Reyes L. D., J. D. Molina G., M. A. Oropeza R. y E. C. Moreno P. (2004) Cruzas dialélicas entre líneas autofecundadas de maíz derivadas de la raza Tuxpeño. Revista Fitotecnia Mexicana 27:49-56

Rimieri P. (2017) La diversidad genética y la variabilidad genética: dos conceptos diferentes asociados al germoplasma y al mejoramiento genético vegetal. Journal of Basic and Applied Genetics 28:7-13.

Romero P. J., F. Castillo G. y R. Ortega P. (2002) Cruzas de poblaciones nativas de maíz de la raza Chalqueño: II. Grupos genéticos, divergencia genética y heterosis. Revista Fitotecnia Mexicana 25:107-115

Sánchez-Hernández C., C. Villanueva-Verduzco, J. Sahagún-Castellanos, J. Martínez-Solís, J. P. Legaria-Solano y M. A. Sánchez-Hernández (2011) Efectos de aptitud combinatoria en híbridos de calabacita tipo Grey Zucchini. Revista Chapingo Serie Horticultura 17:89-103.

Senapati A. K., P. Prajapati and A. Singh (2013) Genetic variability and heritability studies in Gerbera jamesonii Bolus. African Journal of Agricultural Research 8:5090-5092.

Singh S., D. Dhyani, A. Kumar Yadav and S. Rajkumar (2011) Flower colour variations in gerbera (Gerbera jamesonii) population using image analysis. Indian Journal of Agricultural Sciences 81:1130-1136

SNICS, Servicio Nacional de Inspección y Certificación de Semillas (2017) Gaceta oficial de los derechos de obtentor de variedades vegetales. Secretaría de Agricultura, Ganadería, Desarrollo Rural, Pesca y Alimentación. Año 11, Número 27. Ciudad de México. $97 p$

Sprague G. F. and L. A. Tatum (1942) General vs. specific combining ability in single crosses of corn. Journal of the American Society of Agronomy 34:923-932. 